\title{
Population expansions shared among coexisting bacterial lineages are revealed by genetic evidence
}

Comparative population studies can help elucidate the influence of historical events upon current patterns of biodiversity among taxa that coexist in a given geographic area. In particular, comparative assessments derived from population genetics and coalescent theory, have been used to investigate population dynamics of bacterial pathogens to understand disease epidemics. In contrast, and despite the ecological relevance of nonhost associated and naturally occurring bacteria, there is little understanding of the processes determining their diversity. Here we analyzed patterns of genetic diversity in coexisting populations of three genera of bacteria (Bacillus, Exiguobacterium, and Pseudomonas) that are abundant in the aquatic systems of the Cuatro Cienegas Basin, Mexico. We tested the hypothesis that a common habitat leaves a signature upon the genetic variation present in bacterial populations, independent of phylogenetic relationships. We used multilocus markers to assess genetic diversity and 1) performed comparative phylogenetic analyses, 2) described the genetic structure of bacterial populations, 3) calculated descriptive parameters of genetic diversity, (4) performed neutrality tests, and 5) conducted coalescent-based historical reconstructions. Our results show a trend of synchronic expansions across most populations independent of both lineage and sampling site. Thus, we provide empirical evidence supporting the analysis of coexisting bacterial lineages in natural environments to advance our understanding of bacterial evolution beyond medical or health-related microbes. 
3 Morena Avitia ${ }^{1}$, Ana E. Escalante ${ }^{2}$, Eria A. Rebollar ${ }^{1,3}$, Alejandra Moreno-Letelier $^{4}$, Luis E.

4 Eguiarte $^{1}$ and Valeria Souza ${ }^{1 *}$

$5{ }^{1}$ Departamento de Ecología Evolutiva, Instituto de Ecología, Universidad Nacional Autónoma de

6 México, Apartado Postal 70-275, México DF 04510, México.

$7 \quad{ }^{2}$ Departamento de Ecología de la Biodiversidad, Laboratorio Nacional de Ciencias de la

8 Sostenibilidad, Instituto de Ecología, Universidad Nacional Autónoma de México, Apartado

9 Postal 70-275, México DF 04510, México.

$10{ }^{3}$ Biology Department, James Madison University, Bioscience Building 2001, MSC 7801,

11 Harrisonburg VA, USA

$12{ }^{4}$ Division of Biology, Imperial College London, Silwood Park Campus, SL5 7PY Ascot,

13 Berkshire, UK

14 Key words: Bacterial Population Genetics, Coalescence, Cuatro Cienegas, Historical

15 Demography, MLST

16 *Corresponding author: souza@unam.mx

17 ABSTRACT 
18 Comparative population studies can help elucidate the influence of historical events upon current

19 patterns of biodiversity among taxa that coexist in a given geographic area. In particular,

20 comparative assessments derived from population genetics and coalescent theory have been used

21 to investigate population dynamics of bacterial pathogens to understand disease epidemics. In

22 contrast, and despite the ecological relevance of non-host associated and naturally occurring

23 bacteria, there is little understanding of the processes determining their diversity. Here we

24 analyzed patterns of genetic diversity in coexisting populations of three genera of bacteria

25 (Bacillus, Exiguobacterium, and Pseudomonas) that are abundant in the aquatic systems of the

26 Cuatro Cienegas Basin, Mexico. We tested the hypothesis that a common habitat leaves a

27 signature upon the genetic variation present in bacterial populations, independent of phylogenetic

28 relationships. We used multilocus markers to assess genetic diversity and 1) performed

29 comparative phylogenetic analyses, 2) described the genetic structure of bacterial populations, 3)

30 calculated descriptive parameters of genetic diversity, (4) performed neutrality tests, and 5)

31 conducted coalescent-based historical reconstructions. Our results show a trend of synchronic

32 expansions across the studied populations independent of both lineage and sampling site. Thus,

33 we provide empirical evidence supporting the analysis of coexisting bacterial lineages in natural

34 environments to advance our understanding of bacterial evolution beyond medical or health-

35 related microbes. 
INTRODUCTION

37 The present-day distribution of biodiversity is a consequence of the evolutionary dynamics of

38 populations and the history of the regions in which they occur (Lomolino, Riddle \& Brown,

39 2006). Lineage diversification results from interactions between the intrinsic biological

40 constraints of organisms and extrinsic environmental factors (Dawson, 2012). The demographic

41 patterns of coexisting populations have often been analyzed in the context of the history of a

42 region to better understand how the evolution of resident lineages has been affected by their

43 environment (Lessa, Cook, and Patton 2003; Carnaval and Moritz 2008; Ramírez-Barahona and

44 Eguiarte 2013; Chan, Schanzenbach, and Hickerson 2014; Hope et al. 2014). The analysis of

45 genetic traits through the application of coalescent theory has provided evidence of the impacts of

46 past geological and environmental events on the demographic history of resident species, such as

47 the effect of Quaternary glaciations upon the distribution of animal species in North America

48 (Lessa, Cook, and Patton 2003; Hope et al. 2014).

49 It is clear that patterns of biodiversity across phylogenetically distinct taxa may be

50 influenced by shared historical factors (Chan, Schanzenbach, and Hickerson 2014; Hope et al.

51 2014). Despite the importance of microorganisms in ecosystems (Allison \& Martiny 2008;

52 Strickland et al., 2009), the scale of the impact of historical factors remains poorly understood

53 and little is known about the population dynamics of natural microbial populations, which has

54 greatly hindered our understanding of the processes determining diversity. Most studies on

55 microbial population dynamics have analyzed demographic patterns of bacterial pathogens,

56 undertaken primarily to understand disease epidemics and population expansion events of human

57 pathogens (Pybus et al., 2001; Wirth et al., 2007; Tazi et al., 2010). Studies of demographic

58 trends in natural bacterial populations have been scant (Guttman, Morgan \& Wang, 2008), due in

59 part to limited sampling of populations from different lineages at similar temporal and geographic

60 scales. 
61 In this context, the Cuatro Cienegas Basin (CCB) harbors high levels of bacterial

62 diversity, arguably due to environmental variation (e.g. salinity) across the aquatic systems within

63 the basin (Cerritos et al. 2011). However, there is no conclusive evidence that identifies

64 environmental conditions or geographic distance as predictors for the presence of certain

65 bacterial groups (Escalante et al., 2008; Rebollar et al., 2012). Thus the influence of shared

66 historical factors in population dynamics could be a plausible explanation for the observed

67 diversity in $\mathrm{CCB}$. A collection of coexisting bacterial isolates from the aquatic systems in this

68 area has been built for over a decade (Cerritos et al., 2011; Rebollar et al., 2012; Rodríguez-

69 Verdugo et al., 2012) and represents a unique opportunity to investigate historical population

70 patterns of coexisting bacterial lineages in a natural setting.

71 In the present work, we tested the hypothesis that a shared history in the CCB region has

72 left common genetic signatures across phylogenetically diverse microbial populations. Using

73 population genetics and coalescent-based approaches, we assessed the population history of

74 lineages from two closely related genera of Firmicutes, Bacillus and Exiguobacterium, as well as

75 lineages of Gammaproteobacteria from the genus Pseudomonas. The selected lineages are all

76 common in the natural setting of the $\mathrm{CCB}$ aquatic system, and we anticipated that the genetic

77 variation present among coexisting lineages would reveal a signature of common historical

78 dynamics independent of phylogenetic relationships. Multilocus sequence typing (MLST)

79 markers were used to 1) perform comparative phylogenetic analyses, 2) describe the genetic

80 structure of bacterial populations, 3) calculate descriptive parameters of genetic diversity, (4)

81 perform neutrality tests, and 5) conduct historical demography reconstructions.

82 Consistent with our hypothesis, we identified a common trend of expansion in the studied

83 populations over a similar time frame that occurred independent of phylogenetic relationships.

84 These results provide empirical evidence that analyzing coexisting bacterial lineages in natural

85 environments can advance our understanding of bacterial evolution, beyond medical or health- 
related species.

\section{MATERIALS \& METHODS}

\section{Study sites and sampling}

89 Surface water and sediment samples were collected between 2003 and 2009 (Rodríguez-Verdugo

90 et al., 2012; Rebollar et al., 2012) from four aquatic systems within the CCB: Churince (C), Los

91 Hundidos (H), Mesquites (M), and Pozas Azules (Pa). Pseudomonas isolates were collected only

92 from C. All isolates were obtained using previously described methods (Rebollar et al., 2012). All

93 samples were collected under the "Vida Silvestre" permit 0531 FAUT-0230 granted by the

94 Mexican government agency: “Comisión Nacional de Áreas Protegidas” (CONANP).

95 Water and sediment samples were diluted at $1: 10 ; 1: 100 ; 1: 1,000 ;$ and $1: 10,000$ using

96 saline solution $(1 \% \mathrm{NaCl})$. Subsequently, $200 \mu \mathrm{l}$ of each dilution was plated using either marine

97 agar (Difco 2216) for Firmicutes or GSP agar (Kielwein, 1971) for Pseudomonas. Firmicutes

98 isolates were incubated for $24 \mathrm{~h}$ at $37^{\circ} \mathrm{C}$; Bacillus and Exiguobacterium colonies were pale or

99 bright orange, respectively. All orange colonies were purified by single-colony isolation.

100 Pseudomonas isolates were incubated for $48 \mathrm{~h}$ at $30^{\circ} \mathrm{C}$. Pseudomonas colonies were selected

101 based on a change in the color of the GSP media to purple. All isolates were stored at $-80^{\circ} \mathrm{C}$ in $10220 \%(\mathrm{w} / \mathrm{v})$ glycerol.

\section{Molecular markers and sequencing}

104 DNA was extracted from all isolates using a DNAeasy Tissue Kit (Qiagen, Valencia CA, USA),

105 following the manufacturer's instructions. The $16 S r R N A$ gene was amplified and sequenced 106 using 27F and 1492R primers, according to the conditions described by Lane (Lane 1991). PCR

107 products were sequenced and used to identify isolates. Once a strain was identified as Bacillus, 
108 Exiguobacterium or Pseudomonas, we amplified and sequenced a set of housekeeping genes

109 commonly used for MLST (Cerritos et al., 2011; Yamamoto et al., 2000; Sarkar \& Guttman,

110 2004; Rodrigues et al., 2006). Genus-specific PCR primers (Table S1) were used. All PCR

111 products were sequenced at the University of Washington's High Throughput Genomics Center.

112 Sequences were deposited in the GenBank database with the following accession numbers:

113 Bacillus citC (KC900996-KC901178); Bacillus gltx (JQ241465-JQ241624); Bacillus hsp70

114 (KC901179-KC901361); Bacillus recA (JQ247793-JQ247952); Bacillus spo0A (KC901362-

115 KC901540); Exiguobacterium citC (JF916988-JF917080, JF952020-JF952109);

116 Exiguobacterium hsp70 (JF952111-JF952292); Exiguobacterium recA (JF952293-JF952475);

117 Exiguobacterium rрoB (JF952476-JF952658); Pseudomonas acnB (KC953704-KC953753);

118 Pseudomonas gyrB (KC920532-KC920576); Pseudomonas recA (KC961435-KC961462);

119 Pseudomonas rpoD (KC920481-KC920531).

\section{Phylogenetic reconstructions}

121 The complete sequences of the 16S rRNA gene and partial sequences of the MLST genes were

122 edited and aligned using BioEdit (Hall, 1999). Representative $16 S$ rRNA sequences for the three

123 genera were obtained from GenBank and were included in the alignments as references (see

124 Figure S1a-c for accession numbers).

125 We calculated the Pairwise Homoplasy Index $\left(\Phi_{\mathrm{W}}\right)$ using the "PHI test recombination"

126 function implemented in SplitsTree4 (Huson and Bryant 2006) to verify clonality for all lineages

127 as has been reported for Bacillus, Exiguobacterium and Pseudomonas (Roberts and Cohan 1995;

128 Spiers, Buckling, and Rainey 2000; Rebollar et al. 2012). Since all lineages are clonal (Table S2),

129 we were able to use concatenated data sets for phylogenetic reconstruction and population 130 genetics analyses.

131 For all genera, maximum likelihood (ML) phylogenies were constructed using (i) $16 \mathrm{~S}$ 
$132 r R N A$ sequences and (ii) concatenated alignments of MLST sequences. The program jModelTest

133 v. 2.1.3 was used to determine the best nucleotide substitution model for each alignment (Posada,

134 2008; Darriba et al., 2012). For the $16 S$ rRNA gene, , TPM2uf $+\mathrm{I}+\mathrm{G}$, TIM1+I+G and GTR $+\mathrm{I}+\mathrm{G}$

135 were the substitution models selected for, Bacillus, Exiguobacterium and Pseudomonas,

136 respectively. GTR $+\mathrm{I}+\mathrm{G}$, TIM2 $+1+\mathrm{G}$, and GTR $+\mathrm{I}+\mathrm{G}$ were the models selected for the Bacillus,

137 Exiguobacterium, and Pseudomonas concatenated MLST loci alignments, respectively.

138 Phylogenetic relationships were reconstructed using PhyML v.3.0 (Guindon \& Gascuel, 2003),

139 with the corresponding DNA substitution models, tree improvement was carried out by Subtree

140 Pruning and Regrafting, and branch support was evaluated by 1,000 bootstrap pseudo-replicates.

\section{Population structure}

142 We defined populations as the minimal study unit in our collection. Populations have to be

143 defined considering both geographic and genetic criteria. Given that it is possible to find

144 populations (genetic pools) that are not site-restricted we investigated population structure by

145 genetic differentiation, which is defined as changes in the frequency distribution of haplotype

146 variants among subpopulations (Hartl and Clark 1997). We estimated pairwise $F_{S T}$ values among

147 sampling sites within lineages. With this approach we defined a single population as all isolates

148 from a lineage that exhibited no significant genetic differentiation as measured by pairwise $F_{S T}$

149 estimates. Pairwise $F_{S T}$ estimates were obtained for each monophyletic lineage across all

150 sampling sites using Arlequin 3.5 (Excoffier \& Lischer, 2010), with 1,000 iterations. For the

151 Pseudomonas isolates, $F_{S T}$ analysis was not performed since all isolates were from the same

152 sampling site.

153 The $F_{S T}$ approach implicitly incorporates geographic location and genetic criteria in the

154 investigation of population structure. However, it is possible that genetic structure exists for each

155 lineage when looking at individual genetic variants within each population. Thus, we investigated 
156 potential substructure within the groups defined by $F_{S T}$ by taking a Bayesian approach

157 implemented in BAPS 6 (Tang et al., 2009). The Bayesian analysis of population structure was

158 performed using the option for linked loci, specifically developed for MLST data (Tang et al.,

159 2009). A maximum number of clusters (K) was set to ten, or equal to the number of individuals if

160 these were fewer than ten. Each analysis was replicated ten times.

161 Once populations were defined, standard measures of nucleotide diversity $(\pi)$ and the

162 mutation parameter Watterson's $\theta$ were estimated, together with neutrality tests (Tajima's $D$, Fu 163 and Li's $\mathrm{F}^{*}$ and $\mathrm{D}^{*}$ and Fu's $\mathrm{F}_{\mathrm{S}}$ ) using DNAsp v4.1 (Rozas et al., 2003). Details of these 164 calculations can be found as Supplementary material.

\section{Historical population dynamics}

166 We performed an Extended Bayesian Skyline Plot (EBSP) analysis as implemented in Beast v.

167 1.7.5 (Drummond \& Rambaut 2007; Heled \& Drummond 2008). The EBSP infers past 168 population dynamics from a sample of contemporary sequences, taking into account the 169 genealogical stochasticity of the coalescent (Ho \& Shapiro, 2011; Drummond et al., 2005).

170 Additionally, this method does not depend on a specific a priori demographic model, but infers

171 the number of population size changes directly from the data. As a result, it provides a measure of

172 statistical credibility of the inferred number of population size changes compared to the

173 alternative of constant population size (Heled \& Drummond 2008).

174 For the EBSP analysis, we used all MLST genes, a strict molecular clock, and the same

175 nucleotide substitution models used for phylogenetic reconstruction to estimate changes in

176 population size for each genetically homogeneous population. The substitution rate used for the

177 two Firmicutes genera (Bacillus and Exiguobacterium) was $7 \times 10^{-9}$ substitutions/per

178 site/generation, obtained from a dated phylogenetic tree (Maughan, 2007). The substitution rate 
used for Pseudomonas was $2.8 \times 10^{-8}$ substitutions/per site/generation, based on an experimental evolution study of P. fluorescens (Barrett, MacLean \& Bell, 2006). All time estimates obtained were expressed in number of generations. Changes in population size were expressed as a function of the product of $\mathrm{N}_{\mathrm{e}}$ and the generation time $\left(N_{e}^{*} t\right)$. All analyses were run for $50-100$ million generations, until adequate mixing was achieved. $10 \%$ of burn-in was removed and the sampling was done every 1000 chains. The rest of the parameters were set according to the guidelines of Heled \& Drummond (2008). Results were analyzed with Tracer v. 1.5 and LogCombiner v. 1.7.5 (Drummond \& Rambaut, 2007).

\section{RESULTS}

To investigate the influence of a common habitat in the genetic variation of bacterial populations, we analyzed MLST data from a collection of isolates across three bacterial genera (Bacillus, Exiguobacterium and Pseudomonas) sampled from water bodies in the geographic region of CCB, Mexico.

\section{The phylogenetic history of CCB lineages}

Phylogenetic reconstructions based on MLST and $16 S$ rRNA sequences were used to assign isolates to monophyletic lineages (Figs. 1 and S1 respectively). MLST phylogenies identified at least two lineages within each genus. We named the lineages according to genus: "B" for Bacillus, "E" for Exiguobacterium, and "P" for Pseudomonas.

Among Bacillus isolates, we identified two clusters, B1 and B2 (Fig. 1a); their closest relatives were B. aquimaris, B. vietnamensis, B. marisflavi, and B. coahuilensis (Fig. S1a). Exiguobacterium isolates clustered into three well-defined groups: E1, E2, and E3 (Fig. 1b). For clusters E1 and E2, the most closely related species was E. aurantiacum, and E. profundum was the closest relative of cluster E3. Pseudomonas isolates were divided into three groups: P1, P2, and P3 (Fig.1c). P1 included the majority of isolates and, according to $16 S$ rRNA sequences, it 
203 was closely related to $P$. oryzihabitans. P2 isolates were closely related to $P$. otitidis and $P$.

204 aeruginosa, while P3 had recently been described as a new species, P. cuatrocienegasensis

205 (Escalante et al., 2009) (Fig. S1c).

\section{Population structure}

207 Populations defined by pairwise $F_{S T}$ values corresponded with sampling-site pairs with non-

208 significant $F_{S T}$ values (Table S3). We named the resulting populations indicating lineage and 209 sampling site as: B1_C, B1_HPa, B1_M, B2_C, B2_MPa, E1_CHPa, E1_M, E2_CH, E2_M,

210 E3_CM, P1_C, P2_C, and P3_C. Further exploration of structure within lineages was performed

211 with BAPS and showed clusters that are shared among sites within lineages. In all cases,

212 differences in frequency distribution of haplotypes (clusters) correspond with the population

213 structure that we identified through pairwise $F_{S T}$ (Fig. 2). Therefore, we delimited populations

214 based on $F_{S T}$ estimates since it incorporates both the geographic and genetic components in the 215 definition of populations.

\section{Population history: population genetics and coalescent approaches}

217 Results of the genetic diversity analyses and neutrality tests (including Fu's $\mathrm{F}_{\mathrm{s}}$ ) are presented in

218 Table S4. Neutrality tests suggested expansion events but were inconclusive. In our data set, most

219 values for Tajima's $\mathrm{D}$ and $\mathrm{Fu} \&$ Li's tests were negative, although non-significant, which is 220 suggestive of expansion events of populations. However, Fu's $F_{S}$, a test that was explicitly

221 designed to evaluate population expansions, was not significant in most cases. Overall, these

222 classic population genetics estimates did not allow for strong conclusions about historical 223 population events.

224 To further explore the influence of historical processes in shaping the diversity of these 225 bacterial populations, we used a coalescent approach. Extended Bayesian Skyline Plot (EBSP) 
analyses of populations (genetic pools) showed signals of expansion in 9 of the 13 populations, of

227 which 4 have statistical support for at least one change in population size (Figure 3; for statistical

228 support values of the population size changes see table S4). Within Bacillus, an expansion

229 (although not statistically supported) was detected in one population (B1_HPa) that included

230 most B1 isolates. Plots for the other two B1 populations (B1_M and B1_C) showed constant

231 population size across generations (Fig. 3). The B2 plots showed statistical support for change in

232 population size in at least one population (B2_MPa) and constant population size in another

233 (B2_C). Within Exiguobacterium, there was evidence of expansion in one of two E1 populations

234 (E1_M), in one of the two E2 populations (E2_CH) and a trend of expansion (not statistically

235 supported) in the only E3 population (E3_CM). Finally, the plots for all Pseudomonas

236 populations displayed signals of expansion, although only P2_C was statistically supported.

237 Overall, a trend of expansion was observed for the studied populations independent of

238 phylogenetic relationships or sampling sites. Moreover, the skyline plots revealed a common time

239 frame for the start of expansions (20,000 to 30,000 generations ago, Fig. 3), regardless of lineage-

240 or locus-specific parameters.

\section{DISCUSSION}

243 Diversity patterns in nature are a consequence of the evolutionary dynamics of populations and

244 the history of the regions in which populations occur (Lomolino, Riddle \& Brown, 2006). A

245 powerful approach to unveil the influence of historical factors is to analyze current genetic

246 variation to reconstruct the demographic history of populations (Lessa, Cook, and Patton 2003;

247 Hope et al. 2014). In microorganisms, genetic studies looking at historical demography have

248 mainly focused on host-associated bacteria to gain insight in disease epidemics (Mhedbi-Hajri et 
249 al. 2013; Wirth et al. 2006; Comas et al. 2013; Holt et al. 2013). However, little is known about

250 population dynamics of non-host associated and naturally occurring bacteria.

251 When analyzing bacterial populations caution should be taken in the delimitation of

252 populations, which has to consider key aspects such as the degree of genetic and ecological

253 diversity. It is also important to consider the molecular resolution at which populations are

254 defined (Kopac and Cohan 2011), the more and more variable loci studied the more populations

255 may be identified, in our case few and highly conserved loci may result in a coarse population

256 delimitation. Nonetheless, to include both genetic and ecological aspects in the delimitation of

257 populations, we defined individual populations for each lineage by analyzing genetic structure

258 across sampling sites. The analysis showed that populations defined by the $F_{S T}$ statistic include

259 different sampling sites with contrasting environmental conditions (Rebollar et al. 2012), which

260 is in accordance with previous work where no evidence of geographic or environmental barriers

261 to dispersal exists (Escalante et al. 2008; Rebollar et al. 2012). We explored the existence of

262 subpopulations within the $F_{S T}$-defined populations following a Bayesian approach implemented

263 in BAPS (Tang et al. 2009), and we found more clusters that subdivided even further the

264 populations defined with $F_{S T}$ estimates. However, genetic diversity within the identified

265 populations ( $\pi=0.00032-0.04698$ and Watterson's $\theta=0.00073-0.047$; Table S4) is in the range of

266 values reported for natural bacterial populations (Roberts \& Cohan 1995; Vos \& Velicer 2006),

267 meaning that the observed diversity is within the range of normal polymorphism in bacterial

268 populations. Overall, $F_{S T}$, BAPS, and diversity estimates support our population delimitation and

269 indicate that we are not including unusually divergent individuals into the studied populations,

270 which may confound the population genetics and expansion estimations. Given that classic

271 population genetics estimates did not allow for strong conclusions about historical population

272 events, we used a coalescent approach to further explore the influence of historical processes. 
273 From this approach, we found evidence showing that common historical events were population

274 expansions that occurred in 9 out of 13 populations (Figure 3). Even more, it is noteworthy that at

275 least one population of each genus presented evidence of expansion that was statistically

276 supported. In clonal and free-living populations of bacteria, evolutionary models predict that

277 populations experience strong fluctuations, either by selective sweeps or by metapopulation

278 dynamics (Fraser et al. 2009; Kopac \& Cohan 2011; Shapiro \& Polz 2014). The results obtained

279 in this study can be explained by these models since the analyzed lineages come from a natural

280 setting and present no evidence of recombination (Table S2; Roberts \& Cohan 1995; Spiers,

281 Buckling, \& Rainey 2000; Rebollar et al. 2012). Without recombination, clonal clusters emerge

282 by random mutation, but can quickly drift to extinction unless they have a major selective

283 advantage in which case their presence could suggest the emergence of new ecologically distinct

284 populations (Shapiro and Polz 2014) or ecotypes (Kopac and Cohan 2011). Nonetheless, given

285 that we do not have conclusive evidence that identifies environmental conditions or geographic

286 distance as predictors for the presence of certain bacterial groups or populations (Escalante et al.

287 2008; Rebollar et al. 2012), the influence of shared historical factors in population dynamics

288 could be a plausible explanation for the observed population expansion events in CCB. Given

289 that we used just a few loci to delimit the studied populations, it should be noted here that we

290 cannot rule out the possibility that the observed expansions may be a reflection of the emergence

291 of closely related populations (undetectable to our molecular markers), and not population

292 expansions in size. This ambiguity could be resolved if more and more variable loci, even

293 complete genomes, are analyzed. Despite the alternative interpretations of the results, we

294 consider notable the fact that we identified the same type of events (either diversification or

295 population expansions) in most populations in the same time frame. 
297 ( 20,000 generations ago). Our estimation of time to the most recent population change is

298 calculated in number of generations. Although generation times may vary considerably among

299 bacterial lineages (Mason, 1934; Errington, Powell \& Thompson, 1965), laboratory observations

300 of growth rates of the lineages included in this study do not suggest major differences in

301 generation times between them, which supports the synchrony argument. In this study, we used

302 previously reported estimates for Firmicutes (Bacillus and Exiguobacterium) and Pseudomonas

303 to parameterize the coalescent simulations. We used the same substitution rate for Bacillus and

304 Exiguobacterium since little information is available for Exiguobacterium and also because

305 Maughan (2007) did not detect considerable rate heterogeneity between sporulating and non-

306 sporulating Firmicutes. In this respect, it should be acknowledged that values of substitution rates

307 are critical for the estimation of the temporal scale for the demographic events that result from

308 ESPB analyses (Ho et al. 2011) and ideally should be directly estimated for the studied sample.

309 We are aware that this could impact the temporal scales of our skyline plots, and be of particular

310 relevance when comparing lineages with different substitution rates, but we do not have any

311 reason to doubt the substitution rates reported in the literature. Further work should try to directly

312 estimate substitution rates for the studied lineages to minimize the possibility of different time

313 scales for the expansion events.

314 Despite the fact that expansions are expected in bacterial populations, the time of such

315 expansions may differ among lineages because they depend on specific traits such as mutation

316 rate, life history, and population structure (Avise, 2000; Avise 2009; Kuo \& Ochman, 2009;

317 Maughan, 2007; Woolfit \& Bromham, 2003). Synchrony of expansions in coexisting populations

318 of different lineages are not expected to be observed unless shared environmental and historical

319 factors have a major influence on population dynamics obscuring evidence of lineage specific 
320 adaptation, as has been observed in different organisms including "human associated" pathogens

321 (Ornelas et al., 2013; Comas et al., 2013; Falush et al., 2003). Synchrony of population

322 expansions supports the argument that current genetic variation may be the result of a major

323 regional event over all populations.

324 Currently, we do not have the necessary information or samples from the appropriate 325 temporal and spatial scales to determine the environmental changes associated with the historical

326 events that influenced population dynamics of the studied lineages. However, we can propose an 327 approximate time span in which these demographic events occurred and hypothesize on the 328 environmental causes behind our observations. Most estimates of generation times for bacteria

329 have been determined in laboratory conditions, which may be considerably shorter than 330 generation times in the wild (for Escherichia coli see (Pierucci, 1972; Ochman, Elwyn \& Moran, 331 1999). Thus, we estimated the approximate absolute time of population expansions based on 332 generation times for aquatic bacteria estimated in conditions that are similar to their natural 333 habitat conditions (approximately between 75 and 300 generations per year; Jannasch, 1969;

334 Hendricks, 1972). We selected a generation time at the lower limit (75 gen/yr), because it is 335 possible that the bacteria in our study may grow slowly, given the extreme limitation of nutrients, 336 in particular phosphorous, found in the CCB aquatic system (Souza et al., 2006; Souza et al., 337 2012; Souza et al., 2008). Thus, if the synchronic event occurred 200-300 years ago for most 338 populations, it is tempting to suggest that the population expansions we observed reflect changes 339 resulting from the recent human activity in the $\mathrm{CCB}$ area. In particular, an increase in 340 anthropogenic activity during the late eighteenth and nineteenth centuries has been reported,

341 primarily centered around agriculture and ranching (Alessio-Robles, 1946) which coincides with 342 the time frame of expansions here found.

\section{CONCLUSIONS}


344 This study investigates historical population events of coexisting but phylogenetically distant

345 bacterial lineages. To our knowledge, this is the first report of a synchronous signature of

346 population expansion that occurred independent of phylogenetic relationships, which is in

347 accordance with our initial hypothesis. Our findings provide strong evidence for the potential of

348 comparative population genetics to address questions about the influence of shared historical

349 factors in population evolutionary processes of naturally occurring non-host associated bacteria.

350 This type of information may be important for natural resources management in the context of the

351 ecosystem services that microorganisms provide.

\section{ACKNOWLEDGMENTS}

353 We thank Dr. Evan Carson, Dr. Michael Travisano, Dr. Daniel Piñero, Dr. Juan Pablo Jaramillo

354 and Dr. Santiago Ramírez-Barahona for their constructive reviews of the manuscript. We also

355 thank Laura Espinosa, Dr. Erika Aguirre and Jaime Gasca-Pineda for technical support during the 356 development of the project.

357 This paper constitutes part of the doctoral research of the first author, who thanks the 358 Doctorado en Ciencias Biomédicas, Universidad Nacional Autónoma de México (UNAM) and 359 acknowledges the scholarship (no. 210335) provided by the Consejo Nacional de Ciencia y 360 Tecnología (CONACyT). 
362

363

364

365

366

367

368

369

370

371

372

373

374

375

376

377

378

379

380

381

382

383

384

385

386

387

388

389

390

391

392

393

394

395

396

397

398

399

400

401

402

Alessio-Robles, M. 1946. Historia Política de La Revolución. Mexico: Botas.

Allison, Steven D, and Jennifer B H Martiny. 2008. "Resistance, Resilience, and Redundancy in Microbial Communities." Proceedings of the National Academy of Sciences USA 105: 11512-11519.

Avise, John C. 2000. Phylogeography: The History and Formation of Species. Cambridge, Massachusetts: Harvard University Press.

_ 2009. "Phylogeography: Retrospect and Prospect." Journal of Biogeography 36: 3-15.

Barrett, Rowan D H, R Craig MacLean, and Graham Bell. 2006. "Mutations of Intermediate Effect Are Responsible for Adaptation in Evolving Pseudomonas Fluorescens Populations." Biology Letters 2 (2) (June 22): 236-8. doi:10.1098/rsbl.2006.0439.

Carnaval, Ana Carolina, and Craig Moritz. 2008. "Historical Climate Modelling Predicts Patterns of Current Biodiversity in the Brazilian Atlantic Forest." Journal of Biogeography 35 (7) (July): 1187-1201. doi:10.1111/j.1365-2699.2007.01870.x.

Cerritos, René, Luis E Eguiarte, Morena Avitia, Janet Siefert, Michael Travisano, Alejandra Rodríguez-Verdugo, and Valeria Souza. 2011. "Diversity of Culturable Thermo-Resistant Aquatic Bacteria along an Environmental Gradient in Cuatro Ciénegas, Coahuila, México." Antonie van Leeuwenhoek 99 (2) (February): 303-318. doi:10.1007/s10482-010-9490-9.

Chan, Yvonne L, David Schanzenbach, and Michael J Hickerson. 2014. "Detecting Concerted Demographic Response across Community Assemblages Using Hierarchical Approximate Bayesian Computation." Molecular Biology and Evolution 31 (9) (June 12): 2501-2515. doi:10.1093/molbev/msu187.

Comas, Iñaki, Mireia Coscolla, Tao Luo, Sonia Borrell, Kathryn E Holt, Midori Kato-Maeda, Julian Parkhill, et al. 2013. "Out-of-Africa Migration and Neolithic Coexpansion of Mycobacterium Tuberculosis with Modern Humans.” Nature Genetics 45 (10) (October): 1176-1182. doi:10.1038/ng.2744.

Darriba, D, G L Taboada, R Doallo, and D Posada. 2012. "jModelTest 2: More Models, New Heuristics and Parallel Computing." Nature Methods 9: 772.

Dawson, Michael N. 2012. "Parallel Phylogeographic Structure in Ecologically Similar Sympatric Sister Taxa." Molecular Ecology 21 (4) (February): 987-1004. doi:10.1111/j.1365-294X.2011.05417.x.

Drummond, a J, a Rambaut, B Shapiro, and O G Pybus. 2005. "Bayesian Coalescent Inference of Past Population Dynamics from Molecular Sequences." Molecular Biology and Evolution 22 (5) (May): 1185-1192. doi:10.1093/molbev/msi103.

Drummond, Alexei J, and Andrew Rambaut. 2007. "BEAST: Bayesian Evolutionary Analysis by Sampling Trees." BMC Evolutionary Biology 7 (January): 214. doi:10.1186/1471-2148-7214.

Errington, B Y F P, E Powell, and N Thompson. 1965. "Growth Characteristics of Some GramNegative Bacteria.” Journal of General Microbiology 39: 109-123.

Escalante, Ana E, Jesús Caballero-Mellado, Lourdes Martínez-Aguilar, Alejandra RodríguezVerdugo, Andrea González-González, Jeiry Toribio-Jiménez, and Valeria Souza. 2009. "Pseudomonas Cuatrocienegasensis Sp. Nov., Isolated from an Evaporating Lagoon in the 
Cuatro Cienegas Valley in Coahuila, Mexico." International Journal of Systematic and Evolutionary Microbiology 59 (Pt 6) (June): 1416-20. doi:10.1099/ijs.0.006189-0.

Escalante, Ana E, Luis E Eguiarte, Laura Espinosa-Asuar, Larry J Forney, Ana M Noguez, and Valeria Souza Saldivar. 2008. "Diversity of Aquatic Prokaryotic Communities in the Cuatro Cienegas Basin." FEMS Microbiology Ecology 65 (1) (July): 50-60. doi:10.1111/j.15746941.2008.00496.x.

Excoffier, Laurent, and Heidi E L Lischer. 2010. "Arlequin Suite Ver 3.5: A New Series of Programs to Perform Population Genetics Analyses under Linux and Windows." Molecular Ecology Resources 10 (3) (May): 564-567. doi:10.1111/j.1755-0998.2010.02847.x.

Falush, Daniel, Thierry Wirth, Bodo Linz, Jonathan K Pritchard, Matthew Stephens, Mark Kidd, Martin J Blaser, et al. 2003. "Traces of Human Migrations in Helicobacter Pylori Populations.” Science (New York, N.Y.) 299 (5612) (March 7): 1582-1585. doi:10.1126/science.1080857.

Fraser, Christophe, Eric J Alm, Martin F Polz, Brian G Spratt, and William P Hanage. 2009. "The Bacterial Species Challenge : Ecological Diversity." Science 323: 741-746.

Fu, Y-X. 1997. "Statistical Tests of Neutrality of Mutations Against Population Growth, Hitchhiking and Background Selection." Genetics 147: 915-925.

Guindon, Stéphane, and Olivier Gascuel. 2003. "A Simple, Fast, and Accurate Algorithm to Estimate Large Phylogenies by Maximum Likelihood.” Systematic Biology 52 (5) (October 1): 696-704. doi:10.1080/10635150390235520.

Guttman, D S, R L Morgan, and P W Wang. 2008. “The Evolution of the Pseudomonads.” In Pseudomonas Syringae Pathovars and Related Pathogens - Identification, Epidemiology and Genomics, edited by M'B Fami, 307-319. Springer Science \& Business Media BV.

Hall, Thomas A. 1999. "BioEdit: A User-Friendly Biological Sequence Alignment Editor and Analysis Program for Windows 95/98/NT." Nucleic Acids Symposium Series 41: 95-98.

Hartl, Daniel L., and Andrew G. Clark. 1997. Principles of Population Geneticd. 3rd ed. Sunderland, Massachussetts: Sinauer Associates, Inc.

Heled, Joseph, and Alexei J Drummond. 2008. "Bayesian Inference of Population Size History from Multiple Loci.” BMC Evolutionary Biology 8 (January): 289. doi:10.1186/1471-21488-289.

Hendricks, C W. 1972. "Enteric Bacterial Growth Rates in River Water." Applied Microbiology 24 (2) (August): 168-174.

Ho, Simon Y W, Robert Lanfear, Lindell Bromham, Matthew J Phillips, Julien Soubrier, Allen G Rodrigo, and Alan Cooper. 2011. "Time-Dependent Rates of Molecular Evolution." Molecular Ecology 20 (15) (August): 3087-101. doi:10.1111/j.1365-294X.2011.05178.x.

Ho, Simon Y W, and Beth Shapiro. 2011. "Skyline-Plot Methods for Estimating Demographic History from Nucleotide Sequences.” Molecular Ecology Resources 11 (3) (May): 423-34. doi:10.1111/j.1755-0998.2011.02988.x.

Holt, Kathryn E, Tran Vu, Thieu Nga, Duy Pham, Ha Vinh, Dong Wook, My Phan, and Vu Tra. 2013. "Tracking the Establishment of Local Endemic Populations of an Emergent Enteric Pathogen." Proceedings of the National Academy of Sciences USA 110 (43): 17522-17527. doi:10.1073/pnas.1308632110/-/DCSupplemental.www.pnas.org/cgi/doi/10.1073/pnas.1308 632110. 
Hope, A G, S Y W Ho, J L Malaney, J A Cook, and S L Talbot. 2014. "Accounting for Rate Variation among Lineages in Comparative Demographic Analyses." Evolution; International Journal of Organic Evolution 68 (9): 2689-2700.

Huson, Daniel H, and D Bryant. 2006. "Application of Phylogenetic Networks in Evolutionary Studies." Molecular Biology and Evolution 23 (2): 254-267.

Jannasch, H W. 1969. "Estimations of Bacterial Growth Rates in Natural Waters." Journal of Bacteriology 99 (1) (July): 156-160.

Kielwein, G. 1971. "Die Isolierung Und Differenzierung von Pseudomonaden Aus Lebensmitteln." Arch Lebensmittelhyg 22: 29-37.

Kopac, Sarah, and Freder M Cohan. 2011. "A Theory-Based Pragmatism for Discovering and Classifying Newly Divergent Bacterial Species." In Genetics and Evolution of Infectious Diseases, edited by Michel Tibayrenc. Elsevier.

Kuo, Chih-Horng, and Howard Ochman. 2009. "Inferring Clocks When Lacking Rocks: The Variable Rates of Molecular Evolution in Bacteria." Biology Direct 4 (January): 35. doi:10.1186/1745-6150-4-35.

Lane, D J. 1991. "16S/23S rDNA Sequencing. Nucleic Acid Techniques." In Bacterial Systematics, edited by E Stackebrandt and M Goodfellow, 115-175. New York, USA: John Wiley \& Sons.

Lessa, Enrique P, Joseph A Cook, and James L Patton. 2003. "Genetic Footprints of Demographic Expansion in North America, but Not Amazonia, during the Late Quaternary." Proceedings of the National Academy of Sciences of the United States of America 100 (18) (September 2): 10331-4. doi:10.1073/pnas.1730921100.

Lomolino, M V, B R Riddle, and J H Brown. 2006. Biogeography. Sunderland, Massachussetts: Sinauer Associates, Inc.

Mason, M M. 1934. "A Comparison of the Maximal Growth Rates of Various Bacteria under Optimal Conditions." Journal of Bacteriology 29: 103-110.

Maughan, Heather. 2007. "Rates of Molecular Evolution in Bacteria Are Relatively Constant despite Spore Dormancy." Evolution; International Journal of Organic Evolution 61 (2) (February): 280-288. doi:10.1111/j.1558-5646.2007.00026.x.

Mhedbi-Hajri, Nadia, Ahmed Hajri, Tristan Boureau, Armelle Darrasse, Karine Durand, Chrystelle Brin, Marion Fischer-Le Saux, et al. 2013. "Evolutionary History of the Plant Pathogenic Bacterium Xanthomonas Axonopodis.” PloS One 8 (3) (January): e58474. doi:10.1371/journal.pone.0058474.

Nübel, Ulrich, Janina Dordel, Kevin Kurt, Birgit Strommenger, Henrik Westh, Sanjay K Shukla, Helena Zemlicková, et al. 2010. "A Timescale for Evolution, Population Expansion, and Spatial Spread of an Emerging Clone of Methicillin-Resistant Staphylococcus Aureus." PLoS Pathogens 6 (4) (April): e1000855. doi:10.1371/journal.ppat.1000855.

Ochman, H, S Elwyn, and N a Moran. 1999. "Calibrating Bacterial Evolution." Proceedings of the National Academy of Sciences of the United States of America 96 (22) (October 26): $12638-12643$.

Ornelas, Juan Francisco, Victoria Sosa, Douglas E Soltis, Juan M Daza, Clementina González, Pamela S Soltis, Carla Gutiérrez-Rodríguez, et al. 2013. "Comparative Phylogeographic Analyses Illustrate the Complex Evolutionary History of Threatened Cloud Forests of 
Northern Mesoamerica." PloS One 8 (2) (January): e56283. doi:10.1371/journal.pone.0056283.

Pierucci, O. 1972. "Chromosome Replication and Cell Division in Escherichia Coli at Various Temperatures of Growth." Journal of Baceriology 109 (2): 848-854.

Posada, David. 2008. "jModelTest: Phylogenetic Model Averaging." Molecular Biology and Evolution 25 (7) (July): 1253-1256. doi:10.1093/molbev/msn083.

Pybus, O G, M a Charleston, S Gupta, A Rambaut, E C Holmes, and P H Harvey. 2001. "The Epidemic Behavior of the Hepatitis C Virus." Science 292 (5525) (June 22): 2323-2325. doi:10.1126/science.1058321.

Ramírez-Barahona, Santiago, and Luis E Eguiarte. 2013. "The Role of Glacial Cycles in Promoting Genetic Diversity in the Neotropics: The Case of Cloud Forests during the Last Glacial Maximum." Ecology and Evolution 3 (3) (March): 725-38. doi:10.1002/ece3.483.

Rebollar, Eria a, Morena Avitia, Luis E Eguiarte, Andrea González-González, Lucy Mora, Germán Bonilla-Rosso, and Valeria Souza. 2012. "Water-Sediment Niche Differentiation in Ancient Marine Lineages of Exiguobacterium Endemic to the Cuatro Cienegas Basin." Environmental Microbiology 14 (9) (September): 2323-2333. doi:10.1111/j.14622920.2012.02784.x.

Roberts, M S, and F M Cohan. 1995. "Recombination and Migration Rates in Natural Populations of Bacillus Subtilis and Bacillus Mojavensis." Evolution 49: 1081-1094.

Rodrigues, Debora Frigi, Johan Goris, Tatiana Vishnivetskaya, David Gilichinsky, Michael F Thomashow, and James M Tiedje. 2006. "Characterization of Exiguobacterium Isolates from the Siberian Permafrost. Description of Exiguobacterium Sibiricum Sp. Nov." Extremophiles 10 (4) (August): 285-294. doi:10.1007/s00792-005-0497-5.

Rodríguez-Verdugo, Alejandra, Valeria Souza, Luis E Eguiarte, Ana E Escalante, and Alejandra Rodr. 2012. "Diversity across Seasons of Culturable Pseudomonas from a Desiccation Lagoon in Cuatro Cienegas, Mexico." International Journal of Microbiology 2012 (January): 201389. doi:10.1155/2012/201389.

Rozas, J., J. C. Sanchez-DelBarrio, X. Messeguer, and R. Rozas. 2003. "DnaSP, DNA Polymorphism Analyses by the Coalescent and Other Methods." Bioinformatics 19 (18) (December 10): 2496-2497. doi:10.1093/bioinformatics/btg359.

Sarkar, Sara F, and David S Guttman. 2004. "Evolution of the Core Genome of Pseudomonas Syringae, a Highly Clonal, Endemic Plant Pathogen." Applied and Environmental Microbiology 70 (4): 1999-2012. doi:10.1128/AEM.70.4.1999.

Shapiro, B Jesse, and Martin F Polz. 2014. "Ordering Microbial Diversity into Ecologically and Genetically Cohesive Units." Trends in Microbiology 22 (5) (May): 235-247. doi:10.1016/j.tim.2014.02.006.

Souza, Valeria, Luis E Eguiarte, Janet Siefert, and James J Elser. 2008. "Microbial Endemism: Does Phosphorus Limitation Enhance Speciation?" Nature Reviews. Microbiology 6 (7) (July): 559-64. doi:10.1038/nrmicro1917.

Souza, Valeria, Laura Espinosa-Asuar, Ana E Escalante, Luis E Eguiarte, Jack Farmer, Larry Forney, Lourdes Lloret, et al. 2006. "An Endangered Oasis of Aquatic Microbial Biodiversity in the Chihuahuan Desert." Proceedings of the National Academy of Sciences of the United States of America 103 (17) (April 25): 6565-6570. doi:10.1073/pnas.0601434103. 
533

534

535

536

537

538

539

540

541

542

543

544

545

546

547

548

549

550

551

552

553

554

555

556

557

558

559

560

561

562

563
Souza, Valeria, Janet L Siefert, Ana E Escalante, James J Elser, and Luis E Eguiarte. 2012. "The Cuatro Ciénegas Basin in Coahuila, Mexico: An Astrobiological Precambrian Park." Astrobiology 12 (7) (July): 641-7. doi:10.1089/ast.2011.0675.

Spiers, Andrew J, Angus Buckling, and Paul B Rainey. 2000. "MINI REVIEW The Causes of Pseudomonas Diversity": 2345-2350.

Strickland, Michael S, Christian Lauber, Noah Fierer, and Mark a Bradford. 2009. "Testing the Functional Significance of Microbial Community Composition." Ecology 90 (2) (March): $441-51$.

Tang, J, William P Hanage, C Fraser, and J Corander. 2009. "Identifying Currents in the Gene Pool for Bacterial Populations Using an Integrative Approach." PLoS Computational Biology 5 (8): e1000455.

Tazi, Loubna, Marcos Pérez-Losada, Weiming Gu, Yang Yang, Lin Xue, Keith a Crandall, and Raphael P Viscidi. 2010. "Population Dynamics of Neisseria Gonorrhoeae in Shanghai, China: A Comparative Study." BMC Infectious Diseases 10 (January): 13. doi:10.1186/1471-2334-10-13.

Vos, M, and Gregory J Velicer. 2006. "Genetic Population Structure of the Soil Bacterium Myxococcus Xanthus at the Centimeter Scale." Applied and Environmental Microbiology 72 (5): 3615-3625.

Wirth, Thierry, Daniel Falush, Ruiting Lan, Frances Colles, Patience Mensa, Lothar H Wieler, Helge Karch, et al. 2006. "Sex and Virulence in Escherichia Coli: An Evolutionary Perspective." Molecular Microbiology 60 (5) (June): 1136-1151. doi:10.1111/j.13652958.2006.05172.x.

Wirth, Thierry, Giovanna Morelli, Barica Kusecek, Alex van Belkum, Cindy van der Schee, Axel Meyer, and Mark Achtman. 2007. "The Rise and Spread of a New Pathogen: Seroresistant Moraxella Catarrhalis." Genome Research 17: 1647-1656. doi:10.1101/gr.6122607.7.

Woolfit, Megan, and Lindell Bromham. 2003. "Increased Rates of Sequence Evolution in Endosymbiotic Bacteria and Fungi with Small Effective Population Sizes." Molecular Biology and Evolution 20 (9) (September): 1545-1455. doi:10.1093/molbev/msg167.

Yamamoto, S, H Kasai, D L Arnold, R W Jackson, A Vivian, and S Harayama. 2000. "Phylogeny of the Genus Pseudomonas: Intrageneric Structure Reconstructed from the Nucleotide Sequences of gyrB and rpoD Genes." Microbiology 146 (October): 2385-2394. 
565 Figure 1. MLST phylogenetic reconstructions of isolates collected in the Cuatro Cienegas

566 Basin (CCB), Mexico. Phylogenies were constructed using the maximum likelihood approach

567 and are based on concatenated sequences of at least four MLST loci. (a) Bacillus lineages with $B$.

568 marisflavi as outgroup, and citC, gltX, hsp 70, recA and spo0A loci, (b) Exiguobacterium lineages

569 with E. auranticum as outgroup, and citC, hsp70, recA and rpoB loci, and (c) Pseudomonas

570 lineages with Escherichia coli as outgroup, and $a c n B, \operatorname{gyr} B, \operatorname{rec} A$ and $r p o D$ loci. The scale of the 571 bar represents number of substitutions per site

572 Figure 2. Bayesian Analysis of Population Structure (BAPS) of isolates collected in the

573 Cuatro Cienegas Basin (CCB), Mexico. The analysis was conducted for each studied lineage

574 within each genus (Bacillus= B1, B2; Exiguobacterium=E1, E2, E3; Pseudomonas=P1, P2, P3)

575 using the option for linked loci. The maximum number of clusters (K) was set to 10 , or equal to

576 the number of individuals if these were fewer than 10. Each analysis was replicated 10 times. The

577 columns represent a single multilocus genotype and are identified with distinctive colors

578 corresponding to different genetic clusters. Different populations are graphically denoted with a

579 thick dark line between populations with significant $F_{S T}$ values, which are also shown.

580 Figure 3. Bayesian skyline plots for lineages collected in the Cuatro Cienegas Basin (CCB),

581 Mexico showing the effective population size through time (black line). The shaded area

582 represents $95 \%$ credibility intervals. Labels denote the lineage and sampling sites of each

583 population. B, E, and P stand for Bacillus, Exiguobacterium, and Pseudomonas, respectively. C,

$584 \mathrm{H}, \mathrm{M}$, and Pa stand for the sampling sites Churince, Los Hundidos, Mesquites, and Pozas Azules,

585 respectively. Shaded plots represent populations with constant population sizes. Asterisks indicate

586 populations with statistically supported demographic changes (Table S5). 


\section{Figure 1}

MLST phylogenetic reconstructions of isolates collected in the Cuatro Cienegas Basin (CCB), Mexico.

Phylogenies were constructed using the maximum likelihood approach and are based on concatenated sequences of at least four MLST loci. (a) Bacillus lineages with $B$. marisflavi as outgroup, and citC, gltX, hsp70, recA and spo0A loci, (b) Exiguobacterium lineages with $E$. auranticum as outgroup, and citc, hsp70, recA and rpoB loci, and (c) Pseudomonas lineages with Escherichia coli as outgroup, and $a c n B, g y r B, r e c A$ and $r p o D$ loci. The scale of the bar represents number of substitutions per site.

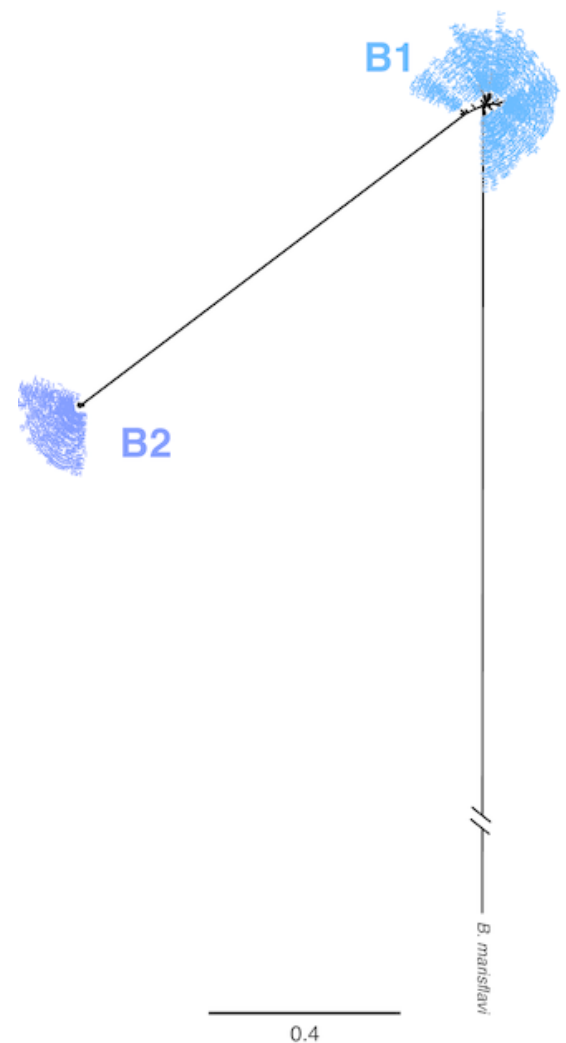

(a) Bacillus

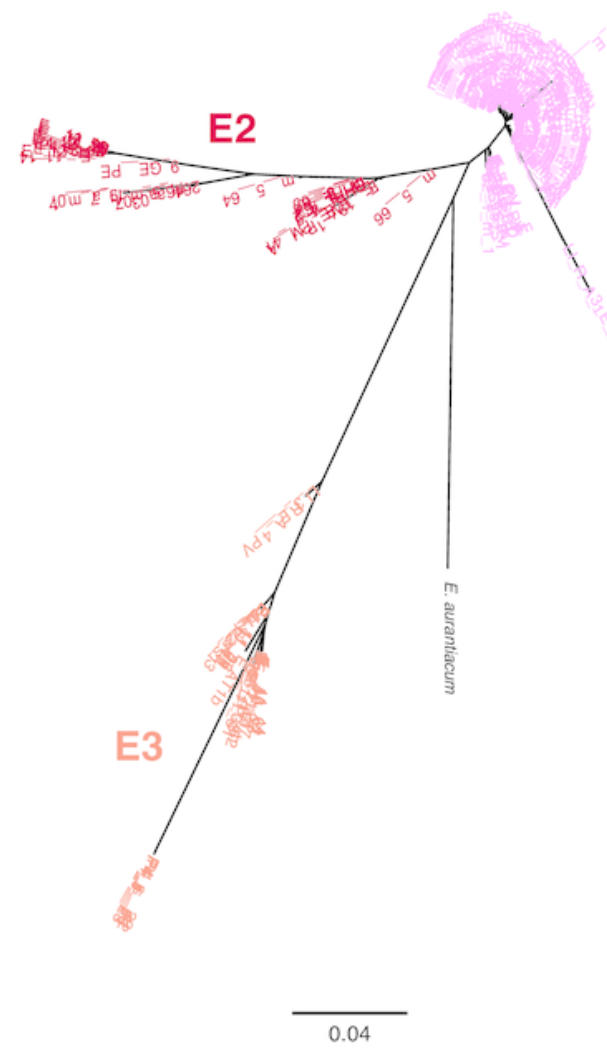

(b) Exiguobacterium

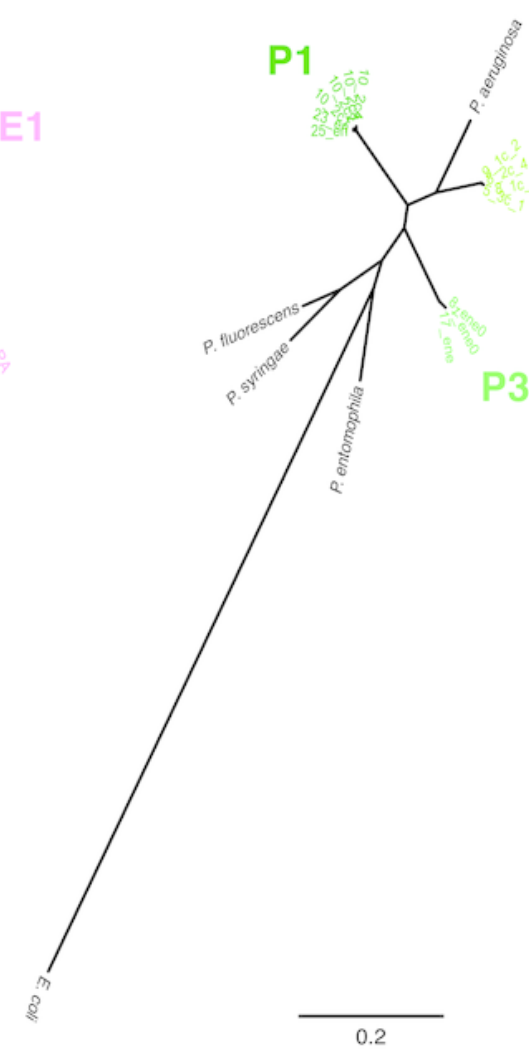

(c) Pseudomonas 


\section{Figure 2}

Bayesian Analysis of Population Structure (BAPS) of isolates collected in the Cuatro Cienegas Basin (CCB), Mexico.

The analysis was conducted for each studied lineage within each genus (Bacillus= B1, B2;

Exiguobacterium=E1, E2, E3; Pseudomonas=P1, P2, P3) using the option for linked loci. The maximum number of clusters $(K)$ was set to 10 , or equal to the number of individuals if these were fewer than 10. Each analysis was replicated 10 times. The columns represent a single multilocus genotype and are identified with distinctive colors corresponding to different genetic clusters. Different populations are graphically denoted with a dark and wide line between populations with significant $F_{S T}$ values, which are also shown. 
a) Bacillus
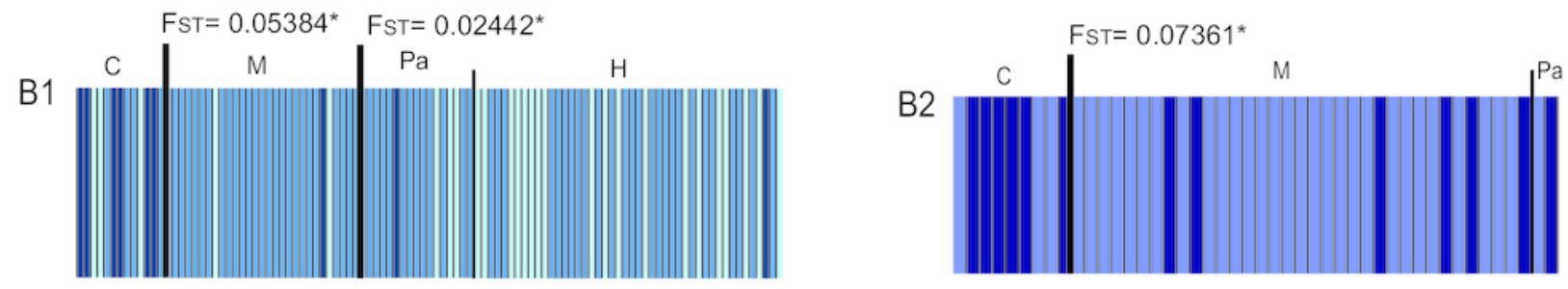

b) Exiguobacterium
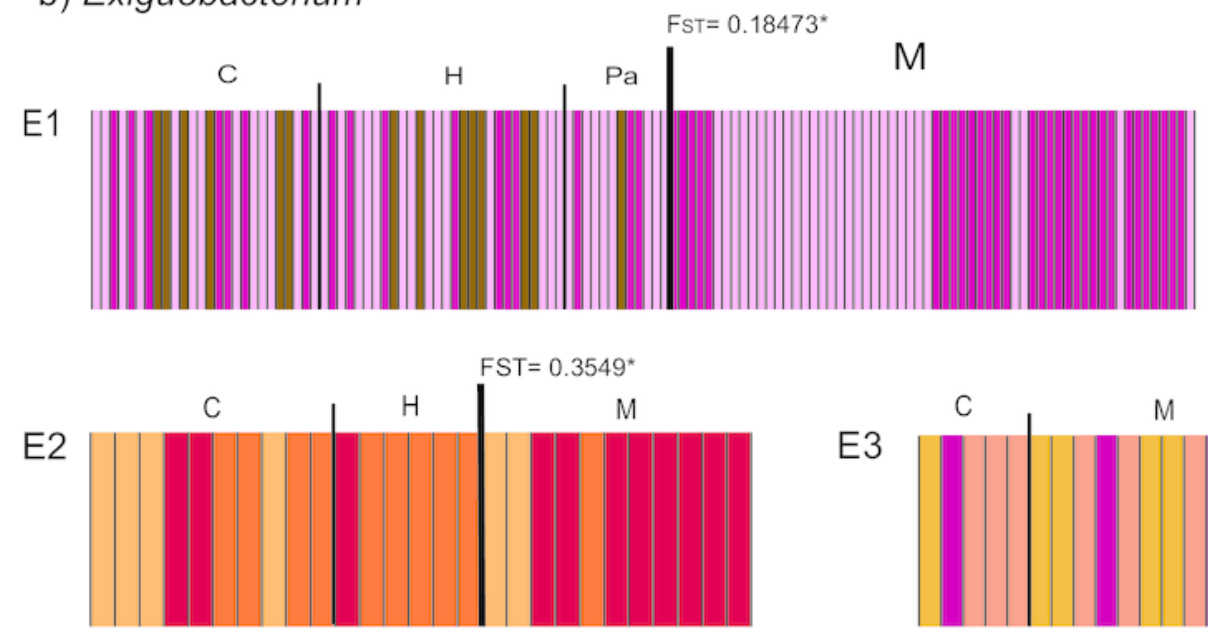

E3

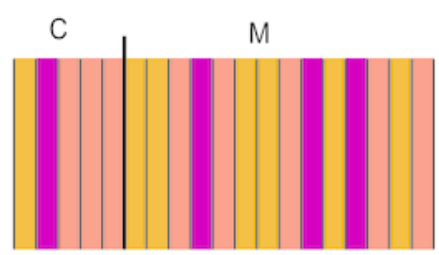

c) Pseudomonas

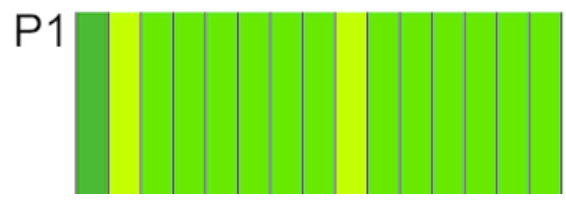

P2

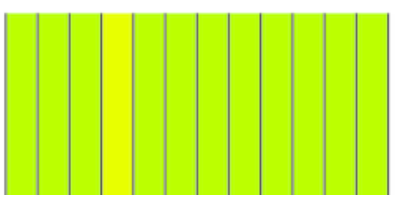

P3

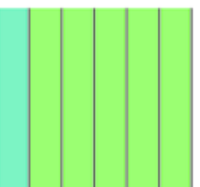




\section{Figure 3}

Bayesian skyline plots for lineages collected in the Cuatro Cienegas Basin (CCB), Mexico showing the effective population size through time (black line). The shaded area represents $95 \%$ credibility intervals. Labels denote the lineage and sampling sites of each population. B, $\mathrm{E}$, and P stand for Bacillus, Exiguobacterium, and Pseudomonas, respectively. C, H, M, and Pa stand for the sampling sites Churince, Los Hundidos, Mesquites, and Pozas Azules, respectively. Shaded plots represent populations with constant population sizes. Asterisks indicate populations with statistically supported demographic changes (Table S5). 

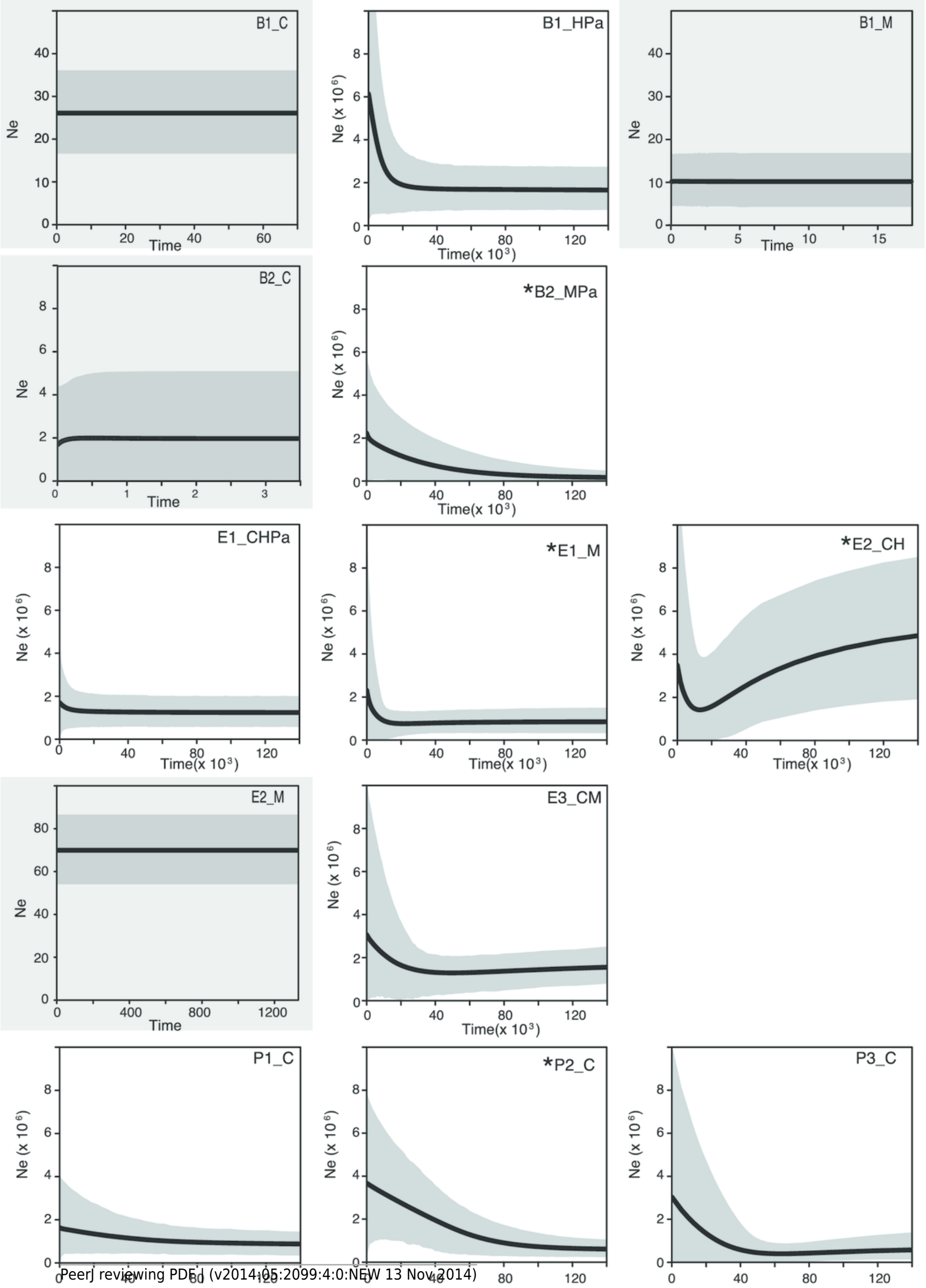

Time $\left(\times 10^{3}\right)$

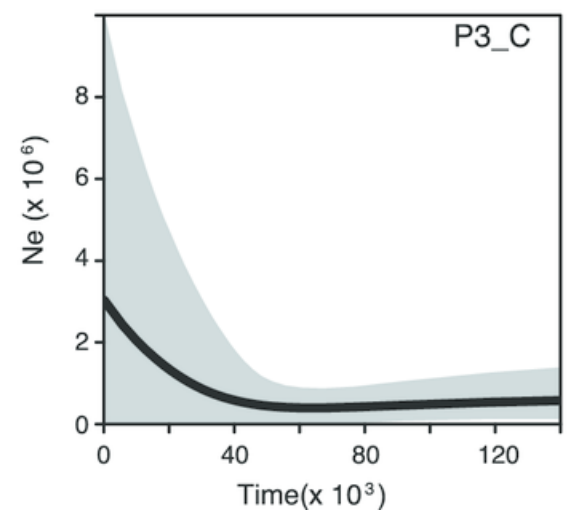

